Gastrointest Endosc. 2014 September ; 80(3): 374-379. doi:10.1016/j.gie.2014.07.001.

\title{
Esophageal diseases
}

\author{
W. Asher Wolf, Evan S. Dellon, and Nicholas J. Shaheen \\ Center for Esophageal Diseases and Swallowing, Division of Gastroenterology and Hepatology, \\ University of North Carolina, Chapel Hill, North Carolina, United States
}

\section{Introduction}

At Digestive Disease Week (DDW) this year (3-6 May, Chicago, Illinois), investigators gathered from around the world to share discoveries and experience in esophageal diseases. Presentations ranged from advances in endoscopic techniques to noninvasive disease stratification and results from long-term cohort studies. This review discusses results from seven seminal studies in esophageal diseases reported at DDW. Although this work is impressive in its scope and potential for clinical impact, selection of these studies as the "most important" is admittedly somewhat arbitrary, as numerous centers contributed a wealth of new information. With that caveat, below we present our review of the most notable abstracts in esophageal diseases from DDW 2014.

\section{Eosinophilic esophagitis}

Patients with eosinophilic esophagitis often require many endoscopies during diagnosis and treatment. A patient undergoing clinical work-up according to consensus guidelines is likely to receive, at the minimum, an esophagogastroduodenoscopy (EGD) at baseline, and another after a trial of proton pump inhibitor (PPI), and a third after starting therapy in order to gauge response [1]. For a patient undergoing dietary elimination therapy with serial food reintroduction, an EGD is typically performed after each food is reintroduced, resulting in an average of nearly five more endoscopies in one recent study [2]. This high number of EGDs results in high costs and increased risk for patients.

At DDW, a group from the Mayo Clinic presented results from a proof-of-concept study using the Cytosponge for minimally invasive evaluation of eosinophilic esophagitis [3]. The Cytosponge is a novel device consisting of a foam sponge compressed into a gelatin capsule, which is attached to a string [4]. Patients swallow the capsule, but the string is kept dangling from the mouth. In the stomach, the capsule dissolves and releases the sponge. The

(C) 2014 American Society for Gastrointestinal Endoscopy. Published by Mosby, Inc. All rights reserved.

Corresponding author Nicholas J. Shaheen, MD, MPH Center for Esophageal Diseases and Swallowing Division of Gastroenterology and Hepatology University of North Carolina School of Medicine CB\#7080 Chapel Hill NC 27599-7080 United States Fax: +1-919-843-2508 nshaheen@med.unc.edu.

Competing interests: None.

Publisher's Disclaimer: This is a PDF file of an unedited manuscript that has been accepted for publication. As a service to our customers we are providing this early version of the manuscript. The manuscript will undergo copyediting, typesetting, and review of the resulting proof before it is published in its final citable form. Please note that during the production process errors may be discovered which could affect the content, and all legal disclaimers that apply to the journal pertain. 
unconstrained sponge is then retrieved by pulling the string, causing the sponge to move retrograde up the esophagus. The sponge collects cells along the entire length of the esophagus as it is pulled through.

Katzka et al. enrolled 20 patients with eosinophilic esophagitis and performed Cytosponge sampling, followed by endoscopy with a routine biopsy protocol to compare the two modalities. Of 16 patients with active eosinophilic esophagitis on the biopsy protocol (>15 eosinophils per high-powered field [eos/hpf]), all had at least $1 \mathrm{eos} / \mathrm{hpf}$ on Cytosponge sampling, and 10 had $>15$ eos/hpf (Fig. 1). Four patients had more eos/hpf on Cytosponge analysis than on biopsy sample analysis, and results from one patient showed eosinophils in the Cytosponge sample but not in the biopsy sample. The $r$ value for the comparison of biopsy and Cytosponge was 0.44 , indicating a strong positive correlation. Spongiosis and basal cell hyperplasia were visible on Cytosponge samples. There were no complications with the use of the Cytosponge technique, even though $75 \%$ of patients had esophageal strictures. Endoscopists assessed the post-sponge esophagus for abrasion damage, and no significant mucosal abrasions were identified from the Cytosponge. Finally, all patients preferred the Cytosponge method to endoscopy.

This study suggests a promising new technology for evaluating eosinophilic esophagitis, with high patient tolerability and a good preliminary safety profile. Given the high cost of endoscopy, and the frequent endoscopies necessary to diagnose and monitor eosinophilic esophagitis by current guidelines, an inexpensive, less onerous method for monitoring the condition of the esophagus is highly desirable. The eosinophil cell count cutoff for the diagnosis of eosinophilic esophagitis will have to be standardized for Cytosponge sampling, as will the cutoff for successful treatment, but this technique may have a future role in the economical and accurate monitoring of the esophagus for response to treatment of eosinophilic esophagitis.

\section{Barrett's esophagus}

\section{Tissue sampling}

Standard biopsy protocols in Barrett's esophagus consist of four-quadrant biopsies at 1-2$\mathrm{cm}$ intervals throughout the length of the Barrett's segment [5]. However, this technique leaves most esophageal tissue unsampled, raising the possibility that dysplastic or cancerous tissue may be missed due to sampling error. The abstract presented by Gross et al. at the Presidential Plenary Session demonstrated the use of wide-area tissue sampling (WATS), a technique that combines brush biopsy with computer-assisted tissue analysis, to evaluate patients with gastroesophageal reflux disease (GERD) and Barrett's esophagus who were receiving care from community gastroenterologists [6].

A total of 2559 patients underwent WATS followed by traditional forceps biopsy. WATS samples were analyzed using a neural network, which sorted over 100000 cells from each WATS sample, identifying the 200 most abnormal cells for pathologist review (Fig. 2). Forceps biopsy samples were analyzed using standard histologic techniques. The cohort was predominantly female $(60 \%)$, with an average age of 55 years and an average Barrett's segment length of $<3 \mathrm{~cm}$. 
Traditional biopsy identified Barrett's esophagus in 377 patients (15\%) and dysplasia in 17 (5\% of Barrett's patients). Adjunctive use of WATS sampling identified an additional 258 Barrett's patients, increasing the diagnostic yield to $25 \%$, and found 10 additional cases of dysplasia and 1 cancer. This represents a 68\% increase in Barrett's esophagus diagnostic yield and a $65 \%$ yield in detection of dysplasia or neoplasia. Whether the sensitivity of forceps biopsy was changed by prior brush biopsy was not evaluated, and it is unclear what gold standard should be used to evaluate sensitivity and specificity of WATS. Notably, these findings are bolstered by the results of a systematic review and meta-analysis that was also presented at this year's DDW, and which found that the number needed to treat with WATS to identify one extra case of Barrett's esophagus ranged from 4 to 11, and that the increased diagnostic yield for dysplasia was 30\% (95\% confidence interval 16\%-55\%) [7].

These results suggest that WATS can produce improved sensitivity compared with forceps biopsy and that WATS can be used successfully in practice, though the question of whether the improved performance is attributable to WATS sampling or neural network analysis remains unanswered. Future questions include the impact of these findings on long-term outcomes of patients with Barrett's esophagus, and whether these findings are generalizable to practices not participating in clinical trials.

\section{Radiofrequency ablation}

The incidence of esophageal adenocarcinoma (EAC) after radiofrequency ablation (RFA) for Barrett's esophagus is poorly described, and most previous studies have included too few cancers to generate reliable estimates of cancer risk after RFA treatment. Investigators from the US RFA Registry evaluated the rate of EAC after RFA and the rate of death from EAC, based on the baseline degree of dysplasia prior to treatment [8].

The US RFA Registry cohort consists of 5521 patients who underwent RFA for treatment of Barrett's esophagus at 132 sites across the United States (approximately three-quarters in private practice and one-quarter in academic practice). Of these patients, 5132 met the inclusion criteria of Barrett's esophagus length $\geq 1 \mathrm{~cm}$ and at least one follow-up visit. Nearly half of the patients enrolled in the Registry (48\%) had nondysplastic Barrett's esophagus at baseline, and another $48 \%$ had dysplasia (21\% low grade [LGD], 19\% high grade [HGD], and $8 \%$ indefinite) (Table 1). The remaining $5 \%$ of patients had intramucosal adenocarcinoma $(4 \%)$ or invasive EAC $(1 \%)$ and were included only in the mortality rate calculation.

Over an average follow-up of 2.6 years, 100 cases of EAC developed: 3 in patients with nondysplastic Barrett's esophagus at baseline ( 0.5 per 1000 person-years), 2 in indefinite dysplasia (2.1 per 1000 person-years), 11 in LGD (3.9 per 1000 person-years), and 84 in patients with HGD (30.2 per 1000 person-years). In patients starting treatment without EAC and receiving at least one RFA treatment, the rate of EAC was 7.8 per 1000 person-years. This represents a decrease of at least $50 \%$ in every histologic grade compared with historical results and meta-analyses $[9,10]$.

A second abstract from the Registry demonstrated that most cancers after RFA were intramucosal (56\%), and that the majority of those were able to achieve complete 
eradication of intestinal metaplasia with further endoscopic therapy (69\%) [11]. In the Registry cohort, 8 of 5521 patients died of EAC, 6 of whom had HGD at baseline and 2 with intramucosal adenocarcinoma at baseline. The overall mortality rate from EAC was 0.6 per 1000 person-years. These results demonstrate that EAC and death from EAC were rare events after treatment with RFA, that the majority of patients who developed EAC after treatment with RFA had HGD at baseline, and that all patients who died of EAC had HGD or intramucosal adenocarcinoma at baseline. Compared with historical controls, EAC incidence was diminished in every histology grade, with a reduction of 50\% among patients presenting with HGD and up to an order of magnitude reduction at lower histologic grades.

\section{GERD}

Electrical stimulation therapy (EST) of the lower esophageal sphincter (LES) is a novel therapy to increase the tone of the LES with the goal of reducing reflux events and esophageal acid exposure. A multicenter group examined patients with GERD that was partially responsive to PPI ( $>5$ point improvement in GERD Health-Related Quality of Life [HRQL] on PPI) [12]. Included patients had an LES end expiratory pressure of $>5 \mathrm{mmHg}$, esophageal acid exposures of at least $5 \%$ of $\mathrm{pH}$-metry time with $\mathrm{pH}<4$, hiatal hernia $<3 \mathrm{~cm}$, and Los Angeles grade of esophagitis lower than grade C [12]. In 33 patients who met these criteria, an electrical stimulator was implanted laparoscopically, with electrodes placed on the serosal surface of the gastroesophageal junction. Stimulation was provided at 30-minute intervals 12 times per day, at $20 \mathrm{~Hz}$ and $5 \mathrm{mAmp}$.

From an on-PPI GERD HRQL median score of 16, patients improved to a median of 5 at 3 months which persisted at 6 months (Fig. 3). Median percentage of time with $\mathrm{pH}<4$ improved from $10 \%$ at baseline to $4 \%$ at 3 months and $5 \%$ at 6 months. Notably, $89 \%$ of patients were off PPIs at 6 months. There was a relatively high frequency of adverse events, with 89 adverse events occurring in 27 patients. However, none were unanticipated, and most were typical of a surgical implant procedure, including incisional discomfort and postoperative nausea. A total of 36 adverse events were related to the device, procedure, or therapy. There were two serious adverse events: a small-bowel perforation during implantation and an atrioventricular re-entrant tachycardia requiring ablation (described as not related to the device or procedure). Other adverse events included pocket pain and dysphagia.

Although these results demonstrate impressive effectiveness in reducing symptoms, acid exposure, and PPI use, additional investigation is required to determine the role of this device in clinical practice. Given the high prevalence of GERD that is incompletely responsive to PPI therapy, alternative therapies for this group are desirable, and early data on this procedure suggest that a device such as the one investigated here may be a viable alternative.

\section{Functional heartburn and esophageal hypersensitivity}

Antidepressants, particularly tricyclics, have broad use in functional gastrointestinal disorders, but the utility of these medications in functional esophageal disorders has been under-studied. A group from Thailand conducted a randomized, double-blind, placebo- 
controlled trial to assess the effectiveness of imipramine for esophageal hypersensitivity and functional heartburn [13].

The authors recruited 83 patients with EGD and 24-hour impedance $\mathrm{pH}$ monitoring consistent with esophageal hypersensitivity or functional heartburn. Patients were randomly assigned to 8 weeks of imipramine $25 \mathrm{mg}$ at bedtime or placebo. The authors assessed GERD score and quality of life (measured using the Short Form (36) Health Survey) at baseline and at 4 and 8 weeks of therapy. There was no difference at baseline between the imipramine $(n=43)$ and placebo $(n=40)$ groups on demographic or disease characteristics.

A total of 67 patients completed the trial. Eight discontinued the medication due to perceived side effects (six in the imipramine group, two in the placebo group), and eight did not adhere to the protocol. In an intention-to-treat analysis, there was no difference in response rates, with $37 \%$ of patients in both groups achieving the primary end point of a $50 \%$ decrease in GERD score at 8 weeks compared with baseline. There was no difference in response based on esophageal hypersensitivity or functional heartburn. There was also no difference in quality of life by intention-to-treat analysis. Constipation was more common in the imipramine group (51\% vs. $22 \% ; P=0.01$ ). These results, derived from a well-designed study, show no benefit and some harm - in the form of anticholinergic side effects - from the use of imipramine compared with placebo for the treatment of esophageal hypersensitivity and functional heartburn. Given the common use of this class of agents in individuals with functional GERD disorders, these data should make clinicians question their routine use in this clinical setting. This negative study also highlights the need for novel therapeutic options in this difficult-to-treat patient group.

\section{Achalasia}

The optimal management of achalasia remains debated, and surgical myotomy, pneumatic dilation, and endoscopic myotomy have all been proposed as initial strategies. The use of peroral endoscopic myotomy (POEM) for the treatment of achalasia and associated motility disorders has been increasing, though the technique remains somewhat controversial, and awaits widespread acceptance. One of the pioneers of the procedure, Dr. Haruhiro Inoue, and a team from Japan presented data evaluating POEM for the treatment of achalasia and other spastic and hypertensive motility disorders [14].

The authors prospectively enrolled 500 consecutive patients who underwent the procedure between 2008 and 2013. Patients ranged in age from 3 to 87 years, with a mean age of 45 years. Patients had experienced symptoms for 0.4-63 years prior to treatment (mean 11 years). The POEM procedure took an average of 98 minutes, average myotomy length was $14 \mathrm{~cm}$, and no procedure had to be converted to open surgery. No major complications occurred, though notable adverse events occurred in 3\% of patients, and included mucosal injury $(\mathrm{n}=8)$, submucosal hematoma $(\mathrm{n}=3)$, pleural effusion $(\mathrm{n}=2)$, pneumothorax $(\mathrm{n}=$ $1)$, peritonitis $(n=1)$, and bleeding requiring transfusion $(n=1)$. Postprocedure, $95 \%$ of patients had an Eckardt score of $<3$. Overall, $16 \%$ of patients developed symptomatic GERD, but only $5 \%$ required PPI therapy. For 36 patients who were followed for more than 3 years, the average Eckardt score after 3 years was 2.3 compared with 6.6 at baseline. 
These results indicate that POEM is effective and durable when performed at an expert center.

The choice of POEM as a primary modality for the management of achalasia is likely to depend on local expertise in the various forms of management of this disease. These data suggest that tertiary centers with expertise in endoscopic therapy may be able to provide this modality, with results comparable to or better than traditional modes of achalasia management. Issues of training and certification for this procedure will need to be addressed prior to wide implementation of this approach in the United States.

\section{Esophageal squamous cell carcinoma}

Squamous cell carcinoma (SCC) remains the predominant esophageal malignancy of the developing world, and 5-year survival is less than $10 \%$. The incidence of esophageal SCC is high enough in some areas of Asia to warrant endoscopic screening for the disease. Predictors of dysplasia, and markers of neoplastic progression that do not depend on morphological analysis of tissue biopsies are lacking.

In this setting, Redman et al. tested biomarkers for SCC [15]. Through mining of a publically available dataset, researchers identified 800 genes that were overexpressed in SCC compared with normal esophagus with $P<10^{-5}$. Of the top 50 genes, 18 were overexpressed in the stroma or normal esophagus. Researchers used quantitative polymerase chain reaction in samples from 60 normal esophagi and from 30 with SCC, and identified 21 genes that were significantly overexpressed in SCC. They then performed immunohistochemistry in 60 normal esophagi and 30 each with mild, moderate, and severe dysplasia, and SCC in order to assess dysregulated gene expression for the six genes (CHN1, COL3A1, CTSL, TNFAIP3, and TNC) that demonstrated the best discrimination between normal esophagus and SCC. Of these, CHN1 and TNFAIP3 showed increased staining corresponding to increased dysplasia (Fig. 4). Expression of p53 also increased with increasing dysplasia. These data suggest that these three markers, perhaps taken in conjunction with other clinical and endoscopic variables, may be able to stratify patients based on degree of dysplasia and risk for SCC. Although these findings are provocative, in vivo testing of high-risk individuals is still needed.

\section{References}

1. Dellon ES, Gonsalves N, Hirano I, et al. ACG clinical guideline: evidenced based approach to the diagnosis and management of esophageal eosinophilia and eosinophilic esophagitis (EoE). Am J Gastroenterol. 2013; 108:679-92. [PubMed: 23567357]

2. Wolf WA, Jerath MR, Sperry SL, et al. Dietary elimination therapy is an effective option for adults with eosinophilic esophagitis. Clin Gastroenterol Hepatol. 2014 In press. DOI: 10.1016/j.cgh. 2013.12.034. [Epub ahead of print].

3. Katzka DA, Ravi A, Geno DM, et al. Cytosponge evaluation of eosinophilic esophagitis in comparison to endoscopy: accuracy, safety and tolerability. Gastroenterology. 2014; 146(5 Suppl 1):S-16.

4. Kadri SR, Lao-Sirieix P, O'Donovan M, et al. Acceptability and accuracy of a non-endoscopic screening test for Barrett's oesophagus in primary care: cohort study. BMJ. 2010; 341:c4372. [PubMed: 20833740] 
5. American Gastroenterological Association. American Gastroenterological Association medical position statement on the management of Barrett's esophagus. Gastroenterology. 2011; 140:10841091. [PubMed: 21376940]

6. Gross SA, Kaul V, Infantolino A, Smith MS. Esophageal brush biopsy with computer-assisted tissue analysis increases detection of Barrett's esophagus and dysplasia in a multi-site community-based setting. Gastrointest Endosc. 2014; 79:AB401.

7. Gerson LB, Rutenberg M, Scott R. Incremental benefit of computer-assisted brush-biopsy (WATS3D) compared to standard endoscopic biopsy for detection of Barrett's esophagus (BE) and dysplasia: systematic review and meta-analysis. Gastroenterology. 2014; 146(5 Suppl 1):S-307.

8. Wolf WA, Lightdale CJ, Li N, et al. Incidence of esophageal adenocarcinoma in patients with Barrett's esophagus undergoing radiofrequency ablation (RFA). Gastroenterology. 2014; $146(5$ Suppl 1):S-301-302.

9. Wani S, Puli SR, Shaheen NJ, et al. Esophageal adenocarcinoma in Barrett's esophagus after endoscopic ablative therapy: a meta-analysis and systematic review. Am J Gastroenterol. 2009; 104:502-513. [PubMed: 19174812]

10. Sikkema M, De Jonge PJ, Steyerberg EW, Kuipers EJ. Risk of esophageal adenocarcinoma and mortality in patients with Barrett's esophagus: a systematic review and meta-analysis. Clin Gastroenterol Hepatol. 2010; 8:235-244. [PubMed: 19850156]

11. Wolf WA, Pruitt RE, Ertam A, et al. Predictors of esophageal adenocarcinoma in patients with prior radiofrequency ablation for treatment of Barrett's esophagus: results from the U.S. RFA Registry. Gastrointest Endosc. 2014; 79:AB217.

12. Siersema PD, Bredenoord AJ, Conchillo JM, et al. Electrical stimulation therapy (EST) of the lower esophageal sphincter (LES) - an effective therapy for refractory GERD - interim results of an international multicenter trial. Gastroenterology. 2014; 146(5 Suppl 1):S-167.

13. Limsrivilai J, Charatcharoenwitthaya P, Pausawasdi N, Leelakusolvong S. Imipramine for treatment of esophageal hypersensitivity and functional heartburn: a randomized double-blind, placebo-controlled trial. Gastroenterology. 2014; $146(5$ Suppl 1):S-165.

14. Inoue H, Onimaru M, Ikeda H, et al. Clinical results of 500 POEM cases for esophageal achalasia and related diseases in a single institute. Gastrointest Endosc. 2014; 79:AB165.

15. Redman JE, Newton R, Malhotra S, et al. Biomarkers for early detection of esophageal squamous cell dysplasia and carcinoma. Gastroenterology. 2014; 146(5 Suppl 1):S-96. 

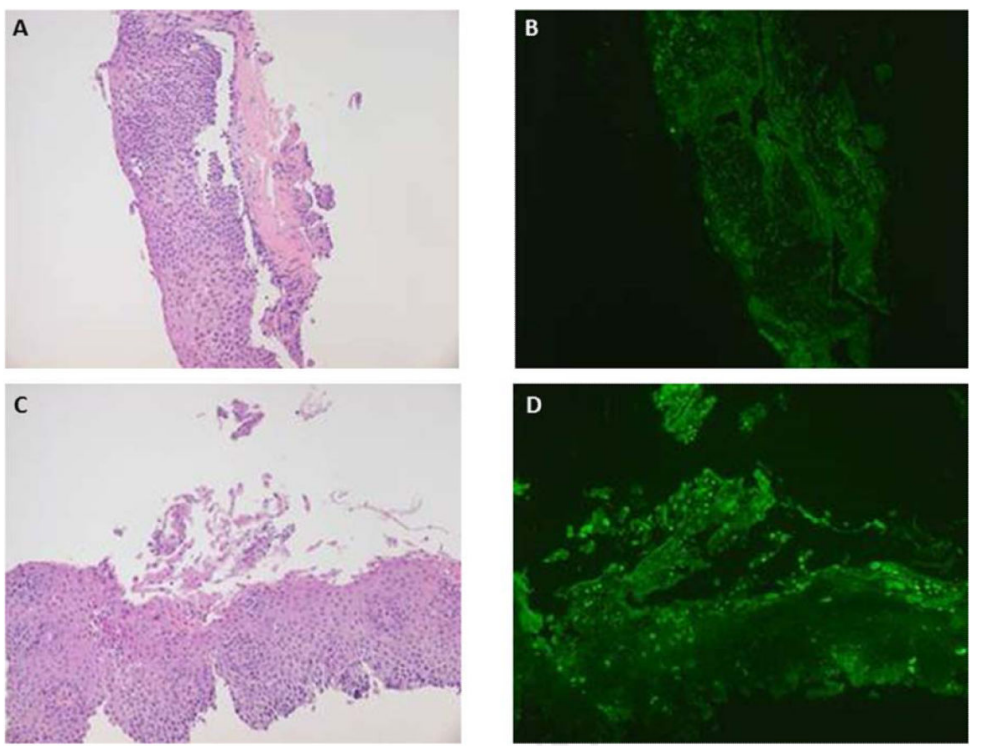

Fig. 1.

Specimens obtained from Cytosponge sampling from two patients, demonstrating the extensive amount of tissue that can be obtained with this technique. a,c Esophageal tissue samples stained with routine hematoxylin and eosin. b,d Markedly increased immunohistochemical staining for eotaxin-derived neurotoxin for the same specimens. Courtesy of David Katzka. 


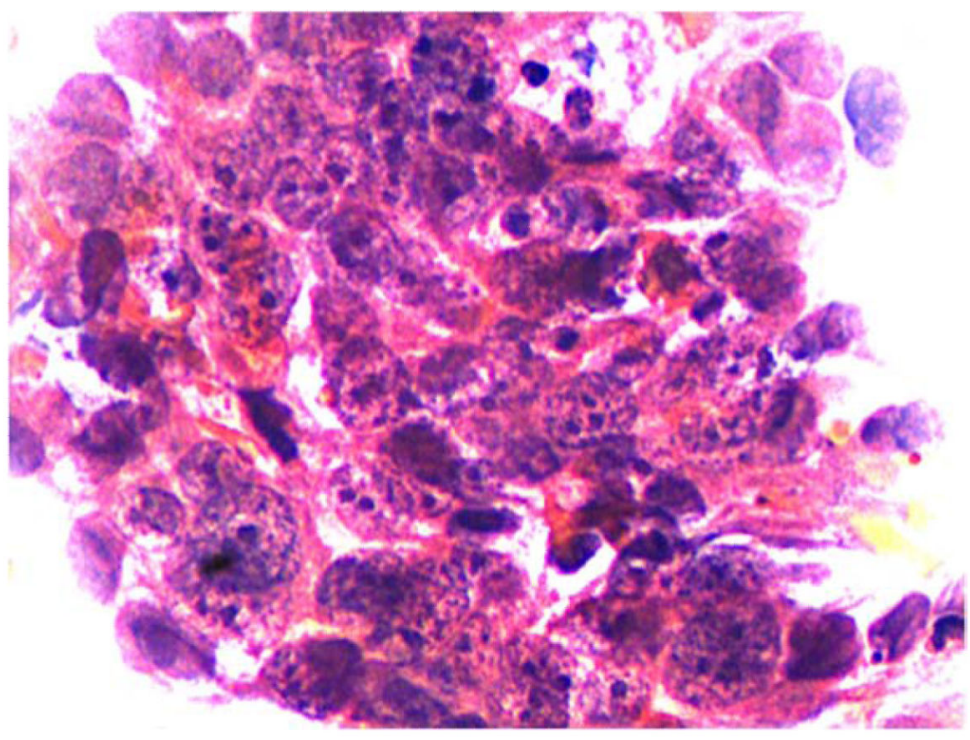

Fig. 2.

Sample obtained by wide-area tissue sampling demonstrating high grade dysplasia with nuclear hyperchromasia, irregular thick nuclear membranes, increased nuclear to cytoplasmic ratio, overlapping nuclei, and loss of nuclear polarity. Courtesy of Seth Gross. 

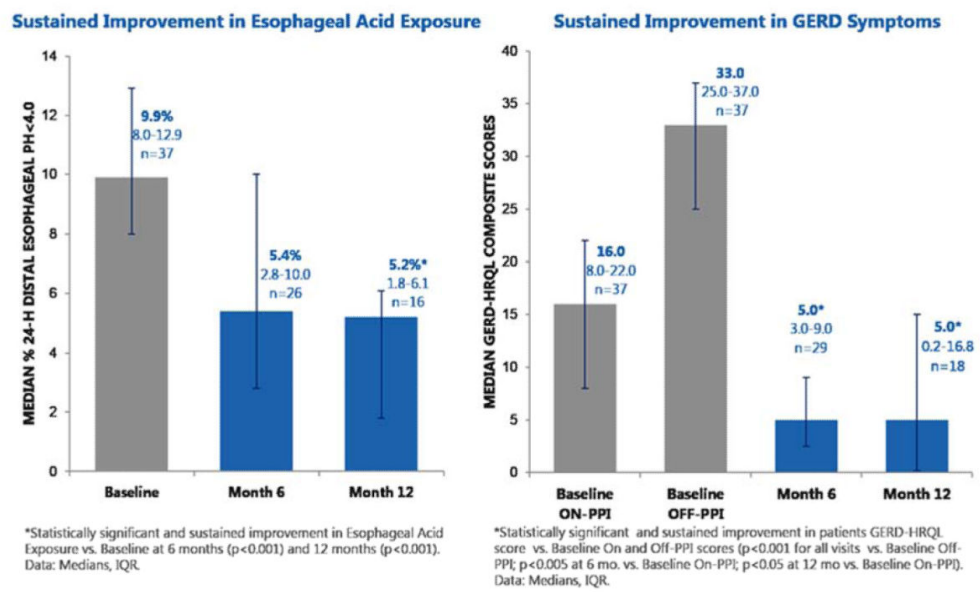

Fig. 3.

a Median hours with distal esophageal $\mathrm{pH}<4$ prior to implantation of electrical stimulator (gray) and at 6 and 12 months post-implantation (blue). b Median gastroesophageal reflux disease health-related quality of life (GERD-HRQL) scores on and off proton pump inhibitor (PPI) (gray), and at 6 and 12 months after implantation (blue). Error bars represent interquartile range. Courtesy of Peter Siersma. 

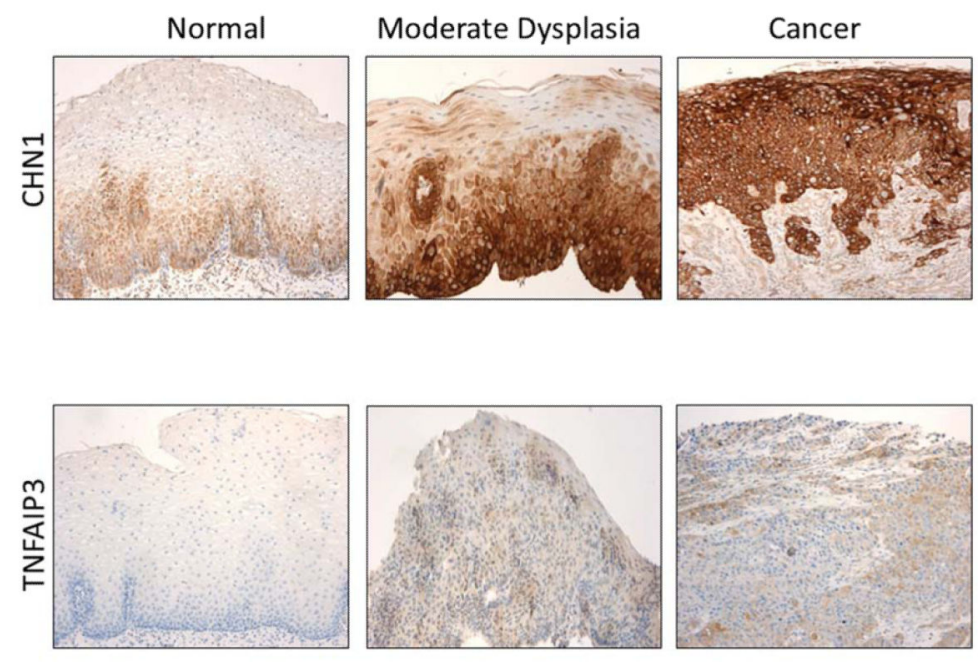

Fig. 4.

Immunohistochemical staining demonstrates increased staining for CHN1 and TNFAIP3 with dysplasia and malignant transformation. Courtesy of Pierre Lao-Sirieix. 


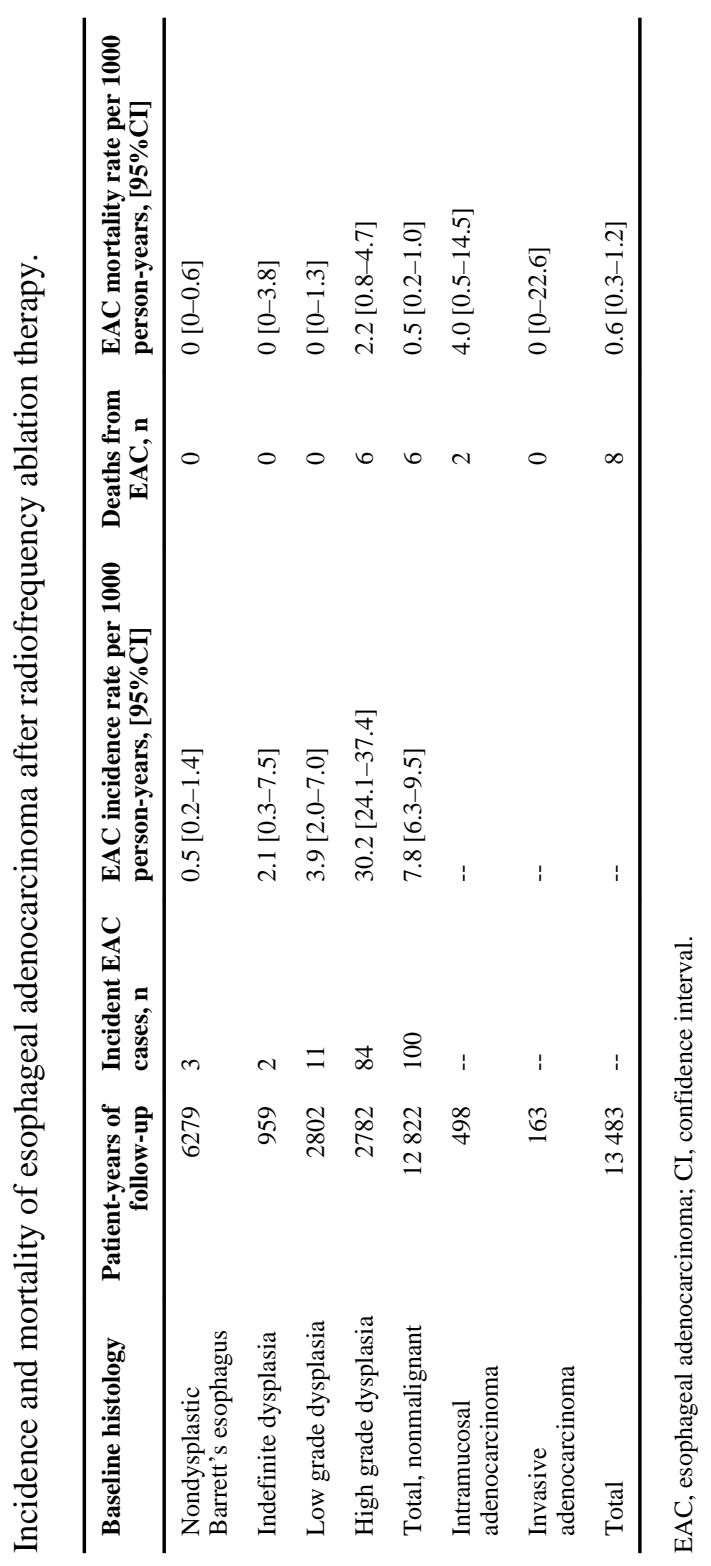

